\title{
An Intelligent UAV based Data Aggregation Strategy for loT After Disaster Scenarios
}

\author{
Xiaoding Wang \\ wangdin1982@163.com \\ College of Mathematics and \\ Informatics, Fujian Normal \\ University \\ Fuzhou, Fujian
}

\author{
Jia $\mathrm{Hu}$ \\ j.hu@exeter.ac.uk \\ University of Exeter \\ Exeter, UK
}

\author{
Hui Lin \\ linhui@fjnu.edu.cn \\ College of Mathematics and \\ Informatics, Fujian Normal \\ University \\ Fuzhou, Fujian
}

\begin{abstract}
The study on data aggregation in Internet of Things (IoT) has drawn a great attention in recent years. Since a large-scale disaster could damage the entire communication network and cut off data aggregation completely, an Intelligent UAV based Data Aggregation Strategy, named (IDAS), is proposed for after disaster scenarios in IoT. Specifically, IDAS first employs an task distribution mechanism to achieve the trade-off between the aggregation ratio and the energy cost. Then, a deep reinforcement learning method is developed for UAV route design to perform corresponding task. Thus, all data are aggregated toward the rescue headquarter by UAV deployment. The simulation results indicate that IDAS has a higher aggregation ratio and a lower energy cost while compared with contemporary strategies.
\end{abstract}

\section{KEYWORDS}

UAV, Data Aggregation, Deep Reinforcement Learning, IoT

\section{ACM Reference Format:}

Xiaoding Wang, Jia Hu, and Hui Lin. 2020. An Intelligent UAV based Data Aggregation Strategy for IoT After Disaster Scenarios. In ACM MobiCom Workshop on Drone Assisted Wireless Communications for $5 G$ and Beyond (DroneCom 20), September 25, 2020, London, United Kingdom. ACM, New York, NY, USA, 5 pages. https://doi.org/10.1145/3414045.3415940

\section{INTRODUCTION}

Internet of Things (IoT) is the interconnection of smart things with sensing, actuation and computing capabilities via the internet. Data collected from smart devices, i.e., smart phones, smart bracelets, smart watchs etc., will be aggregated and analyzed for industrial applications. However, a large-scale disaster could compromise the entire communication network of the IoT, in which deploying UAV will result in the quickest and easiest way to restore a basic communication service in

Permission to make digital or hard copies of all or part of this work for personal or classroom use is granted without fee provided that copies are not made or distributed for profit or commercial advantage and that copies bear this notice and the full citation on the first page. Copyrights for components of this work owned by others than ACM must be honored. Abstracting with credit is permitted. To copy otherwise, or republish, to post on servers or to redistribute to lists, requires prior specific permission and/or a fee. Request permissions from permissions@acm.org.

DroneCom 20, September 25, 2020, London, United Kingdom

(C) 2020 Association for Computing Machinery.

ACM ISBN 978-1-4503-8105-5/20/09.

https://doi.org/10.1145/3414045.3415940 the affected zone. In this paper, we propose an Intelligent Data Aggregation Strategy (IDAS) for IoT using UAV after disasters. Specifically, IDAS consists of a task distribution and a UAV based route design. The details of our contributions are listed as follows. To ensure the data aggregation, IDAS first employs an data aggregation task distribution mechanism. Specifically, this mechanism guarantees that the time gap of visiting the same location should be less than that of filling up the buffer for aggregation ratio improvement. More importantly, the approximate route energy efficiency is introduced to ensure the trade-off between aggregation ratio and energy cost during task distribution. Next, a Deep Reinforcement Learning (DRL) method is developed for each energy efficient UAV route design w.r.t the corresponding data collection task. Thus, all data will be collected and aggregated toward the rescue headquarter through a connected network by UAV deployment. The simulation results indicate that the proposed IDAS has a higher aggregation ratio and a less energy cost while compared with contemporary strategies.

The rest of the paper is organized as follows. Related work is covered in Section 2. The system model is introduced in Section 3. The IDAS is elaborated in Section 4. The validation experiments are presented in section 5 . We conclude this paper in Section 6.

\section{RELATED WORK}

In an after-disaster scenario, a fundamental aspect is how to deploy UAV for data aggregation. In the study of route design, Abbas and Younis [5] utilize convex hulls of components for data aggregation. Similarly, the RCR [3] is proposed to further shorten the vehicle routes by deploying relays. CISIL [1] is develop by exploiting the Delaunay triangulation, in which $k 3$-hyperedges of the 3 -hypergraph are chosen as routes. The LEEF [2] is designed to equalize the energy cost through greedy expansion and optimization successively. Apart from shortening routes, other factors, i.e., data collection rate, buffer size and speed, will potentially affect the aggregation ratio. However, all these factors are neglected by previous works. On the other hand, lots of previous works take energy cost into consideration w.r.t realistic terrains. In [6], stochastic geometry is used to optimize the energy cost. The work proposed in [7] presents a dynamic clustering and routing algorithm to maintain connectivity and achieve energy efficiency in a large scale sensor network. In [10], Senturk et 
al. develop the ReBAT that quantifies terrains to discover the minimum energy cost routes. Wang et al. [9] design a hybrid strategy to achieve data aggregation in realistic environments. Recently, Wang et al. [11] develop a machine learning based strategy for data aggregation. Toyoshima et al. [5] propose Deep Q-Network (DQN) based vehicle simulation systems, which is called in this strategy DQNMDC in this paper for simplicity, considering three-dimensional environment for normal and uniform distributions of events. The efficiency of optimal drone positioning has attracted a lot of interest among researchers and academicians. Zorbas et al. [12] introduces a minimum cost drone location problem. Tuba et al. [13] present a study in which they look into a recent brainstorm optimization algorithm. It aims at finding the optimal positions for static drones in a monitored area such that their coverage is maximized. Shakhatreh et al. [14] talk about finding an optimal position for the UAVs such that the sum of time durations of uplink transmissions is maximized. In [15], Rodriguez et al. compare four studies that have been done on routing and wavelength assignment with the aim of supporting and improving traffic related problems. However, these works are not energy efficient, i.e., the trade-off between aggregation ratio and energy cost is not accomplished.

\section{SYSTEM MODEL}

Consider a damaged IoT network mapped to a graph, in which each node $n_{i}$ represents a survived infrastructures, i.e., a communication tower, with a communication rage $R$, a buffer $B u f_{n_{i}}$ and a data collection rate $D c r_{n_{i}}$. Each component $G_{i}$ exists if a number of survived infrastructure $n_{i}$ s fall into each other's communication range. As the disaster can cause a large-scale damage, i.e., worker injury, factory collapse, communication interruptions etc., the rescue headquarter should restore the communication with injured workers through survived infrastructures. However, most of the time people are unable to communicate with the rescue headquarter because survived infrastructures are overwhelmed by call attempts or their communications with outside world are completely cut off. In these cases, deploying UAVs [16] results in the quickest and easiest way to restore a basic communication service in the affected zone for distress calls or mayday signals aggregation.

As we analysed before, two important factors, namely the aggregation ratio and the energy cost, should be considered during the UAV deployment. Recall that the aggregation ratio relies on the travelling distance that somehow determines the energy cost. However, terrains of realistic environments are dominant for energy cost. That indicates the importance for terrain quantification.

\subsection{Terrain Quantification}

We apply the grid based quantification to measure terrain influences. Specifically, each cell $c$ of the grid is associated with an energy factor $\mathcal{F}$ on a certain terrain as

$$
\mathcal{F}_{c}=\int_{l_{c}} \int_{e_{c}} r_{c},
$$

where $l_{c}, r_{c}$ and $e_{c}$ represent the travelling distance, the risk and the elevation of $c$, respectively. Thus, the energy factor $\mathcal{F}_{T}$ of route $T$ is the sum of that of each sub-route $T_{i}$, which is given by

$$
\mathcal{F}_{T}=\sum_{T_{i} \in T} \sum_{c \in T_{i}} \mathcal{F}_{c}
$$

Accordingly, the energy cost of UAV on route $T$, which is denoted as $\mathbb{E}_{T}^{U A V}$, is then given by

$$
\mathbb{E}_{T}^{U A V}=\mathcal{F}_{T} \times \nu
$$

where $\nu \propto V$. Obviously, $\mathbb{E}_{T}^{U A V}$ is proportional to terrain influences. If an UAV visits a component $G_{i}$ on route $T_{i}$, then all data of which is collected at a specific collection position $p_{i}^{c}$. We then define the energy cost function of a node $n_{j} \in G_{i}$ for data collection as

$$
\mathbb{E}_{n_{j} p_{i}^{c}}^{N o d e}=\kappa \times L_{n_{j} p_{i}^{c}}^{2}
$$

where $\kappa$ is a power related constant and $L_{n_{j} p_{i}^{c}}$ denotes the length of the edge $\left(n_{j} p_{i}^{c}\right)$. Thus, we can deduce that $p_{i}^{c}$ is the centroid of component $G_{i}$ due to $p_{i}^{c}=\arg \min \sum_{n_{j} \in G_{i}} \mathbb{E}_{n_{j} p_{i}^{c}}^{N o \text {. }}$. Then, the energy cost for data collection is given by

$$
\mathbb{E}_{T}^{N o d e}=\sum_{T_{i} \in T} \sum_{C_{i} \in T_{i}} \sum_{n_{j} \in C_{i}} \mathbb{E}_{n_{j} p_{i}^{c}}^{\text {Node }}
$$

Thus, the overall energy cost of route $T$ is then given by

$$
\mathbb{E}_{T}=\mathbb{E}_{T}^{U A V}+\mathbb{E}_{T}^{N o d e} .
$$

Note that reducing energy cost may somehow contradict to aggregation ratio improvement, i.e., a route of less energy cost might require a detour that results in a less aggregation ratio. Thus, we focus on developing an energy efficient data aggregation strategy to achieve the tradeoff between aggregation ratio and energy cost.

\section{THE IDAS APPROACH}

\subsection{Data Aggregation Task Distribution}

Recall that all data of a component $G_{i}$ will be collected while an UAV reached the position $p_{i}^{c}$. That suggests the set $P=\left\{p_{i}^{c}\right\}$ of data collection positions should be partitioned into $N_{m}$ nonoverlapping clusters $C_{i}$ s, each of which is assigned an UAV responsible to collect and aggregate data for corresponding $G_{i}$ s. Otherwise, a component, say $G_{i}$, could be visited by several UAVs such that the time gap of visiting another component $G_{j}$ is enlarged and data is lost due to each node has a limited buffer size. That suggests the time gap should be less or equal to the minimum time of filling up the buffer $B u f_{n_{i}}$ as

$$
\frac{L}{V} \leq \frac{B u f_{n_{i}}}{\operatorname{Dcr}_{G_{i}}}(1+\eta),
$$

where $L$ represents the length of the route, $\eta$ is the tolerable data loss ratio, and $\operatorname{Dcr}_{G_{i}}$ denotes the data collection rate of component $G_{i}$, i.e., $D c r_{G_{i}}=\max _{n_{i} \in G_{i}} D c r_{n_{i}}$. Accordingly, a greedy partitioning algorithm is developed as follows:

Step 1 , construct a Hamilton cycle $H_{P}$ over set $P$ and label each $p_{i}^{c} \in H_{P}$ sequentially along the $H_{P}$, i.e., $H_{P}=$ 
$p_{1}^{c} p_{2}^{c} \ldots p_{n}^{c} p_{1}^{c}$, then repeat Step 2 with a different starting position $p_{j}^{c} \in H_{p}$ each time;

Step 2, sequentially add $p_{i}^{c}$ to $C_{j}$, i.e., $C_{j}=C_{j} \cup\left\{p_{i}^{c}\right\}$, only if $L_{H_{C_{j}}}$ satisfies (7); Otherwise, add current position $p_{k}^{c}$ to $C_{j+1}$ and repeat this step until each $p_{i}^{c} \in H_{P}$ belongs to a certain cluster;

Although a number of partitions are available, the optimal one should take the energy cost into consideration. In order to approximate the real energy cost of a certain partition, we first introduce the probability density functions (pdf) of the distribution of different terrains. From the global perspective, the energy cost for each cell $c$ should consider the pdf of the corresponding terrain $p_{c}$ as

$$
\mathcal{F}_{c}^{\prime}=\int_{l_{c}} \int_{e_{c}} r_{c} p_{c}
$$

Thus, the approximated energy cost $\mathbb{E}_{T}^{\prime}$ is computed utilizing E.q. (2) (5) and (8). Then, we define the Approximated Route Energy Efficiency as the proportion between aggregated data and corresponding energy cost. Obviously, the optimal partition should be the one of the maximal overall approximated energy efficiency. Although, each cluster of the optimal partition is assigned an UAV for data collection and aggregation, in which the energy efficient route within each cluster should be discovered.

\subsection{DRL based Route Design}

We consider the UAV route design in a fully distributed and continuous multi-agent data collection environment. That suggests traditional policy gradient based methods of DRL cannot meet our requirement. For example, DQN can only work well in a limited action space which is discrete, discontinuous and non-distributed, thus it is not suitable for our application scenario. We therefore propose a new solution here. Each UAV $m$ generates an observation $o_{t}^{m}=\left(x_{t}^{m}, y_{t}^{m}, r_{t}^{m}\right)$ which is a part of state $s_{t}$ at each timeslot $t$, and gives it an action $a_{t}^{m}$, then obtains a reward $r_{t}^{m}$ from the environment. After the execution of actions, the environment would change from old state $s_{t}$ to new state $s_{t+1}$. In fact, state, action and reward are three basic components for DRL. Once a state and a set of possible actions are given, and then the goal is to find a policy that maximizes the accumulated reward. In our system, state, action and reward are defined as follows.

1) State Space: State, which is a description of the environment, is denoted as $S=\left\{\left(S_{1}, S_{2}, S_{3}\right)\right\}$ as three channels. We assume that the simulated environment is a map of real terrains. And $S_{1}$ represents a cluster $C^{m}$ of collection positions assigned to a specific UAV $m$, therefore can be defined as $S_{1}=\left\{\left(x^{k, m}, y^{k, m}\right)\right\}_{1 \leq k \leq\left|C^{m}\right|} ; S_{2}=\left\{\left(x_{t}^{m}, y_{t}^{m}\right)\right\}$, where $x_{t}^{m}$, $y_{t}^{m}$ are coordinates of UAV $m$ at timeslot $t ; S_{3}=\left\{n_{t}^{m}\right\}$ is the set of remaining collection position count of $m$ at timeslot $t$, therefore $n_{t+1}^{m}=n_{t}^{m}-1$ if a collection position is visited and the corresponding data is collected at timeslot $t+1$.

2) Action Space: Moving direction $\vartheta_{t}^{m}$ and distance $d_{t}^{m}$ consists of the action set $A=\left\{\left(\vartheta_{t}^{m}, d_{t}^{m}\right) \mid \vartheta_{t}^{m} \in[0,2 \pi], d_{t}^{m} \in\right.$ $\left.\left[0, d_{\max }\right]\right\}$, where $d_{\max }$ is the maximum distance that an UAV can move in a timeslot.

3) Reward: All four parts, namely data collected $b_{t}^{m}$ by timeslot $t$, remaining collection position ratio $n_{t}^{m} /\left|C^{m}\right|$, and energy consumption $\mathbb{E}_{T}$ contribute to the reward of each UAV $m$. Thus, we can compute a reward formulation $r_{t}^{m}$ as:

$$
r_{t}^{m}=\left\{\begin{array}{l}
\frac{1}{\mathbb{E}_{T}}, \text { if } b_{t}^{m}=0 \\
\frac{n_{t}^{m} b_{t}^{m}}{\left|C^{m}\right| \mathbb{E}_{T}}, \text { if } b_{t}^{m}>0,
\end{array}\right.
$$

Thus, the overall reward is $r_{t}=\sum_{m} r_{t}^{m}$. In fact, the reward formulation enable UAVs to be energy efficient in data collection and aggregation.

Each UAV is implemented by 4 DNNs which serves as actor network $\pi\left(o_{t} \mid \theta^{\pi}\right)$, critic network $Q\left(s_{t}, a_{t} \mid \theta^{Q}\right)$ with randomly initialized weights $\theta^{\pi}, \theta^{Q}$ and their two target networks with parameters $\theta^{\pi^{\prime}}=\theta^{\pi}$ and $\theta^{Q^{\prime}}=\theta^{Q}$, where $o_{t}=$ $\left(o_{t}^{1}, \cdots, o_{t}^{N_{m}}\right), a_{t}=\left(a_{t}^{1}, \cdots, a_{t}^{N_{m}}\right)$, and $s_{t}=\left(s_{t}^{1}, \cdots, s_{t}^{N_{m}}\right)$. In each collection round, we initialize environment and obtain the initial state $s_{0} \in S$.

For distributed training process, a group of transitions, i.e., $\langle S, A, R\rangle$, is sampled as mini-batches from each UAV's private buffer. For each UAV $m$, actor target network will give a target action $a_{t}^{m}$ with given observation $o_{t}^{m}$ from a minibatch. Then, critic network $Q$ is updated through minimizing a loss function $L\left(\theta^{Q}\right)$ as:

$$
\begin{gathered}
L\left(\theta^{Q}\right)=E\left[\left(\mathcal{Y}_{t}-Q\left(s_{t}, a_{t}^{1}, \cdots, a_{t}^{N_{m}} \mid \theta^{Q}\right)\right)^{2}\right], \\
\mathcal{Y}_{t}=\gamma Q^{\prime}\left(s_{t+1}, a_{t+1}^{1}, \cdots, a_{t+1}^{N_{m}} \mid \theta^{Q^{\prime}}\right)+r_{t},
\end{gathered}
$$

while we updated actor network, using the gradient as:

$$
\begin{aligned}
& \nabla_{\theta^{\pi}} J \approx E\left[\nabla_{\theta^{\pi}} \pi\left(o \mid \theta^{\pi}\right) \mid o=o_{t}\right. \\
& \left.\quad \nabla_{a} Q\left(s, a^{1}, \cdots, a^{N_{m}} \mid \theta^{Q}\right) \mid a^{m}=\pi\left(o^{m}\right), o=o_{t}\right],
\end{aligned}
$$

where $a=\left(a^{1}, \cdots, a^{N_{m}}\right)$. Note that target networks are two copies of the actor $\pi$ and critic $Q$ networks, but with different weights update rules. Specifically, after updating weights of networks $\pi$ and $Q$, the weights of target networks, $\theta^{Q^{\prime}}$ and $\theta^{\pi^{\prime}}$, are then slowly updated with the original networks weights and update factor $\tau$ to improve the stability of learning, as:

$$
\begin{gathered}
\theta^{Q^{\prime}}=\tau \theta^{Q}+(1-\tau) \theta^{Q^{\prime}}, \\
\theta^{\pi^{\prime}}=\tau \theta^{\pi}+(1-\tau) \theta^{\pi^{\prime}},
\end{gathered}
$$

After adequate training, all the parameters in four DNNs are optimized for data aggregation task.

\section{PERFORMANCE EVALUATION}

In this section, we compare the proposed strategy with baseline approaches considering an after disaster scenario.

\subsection{Simulation Setup}

The performance of IDAS has been validated through extensive simulation experiments which is developed in Python on an Intel Core i5-8250U 1.6 GHZ CPU, 8GB RAM computer. In the simulation, we assume that all devices are 
deployed within $1000 m \times 1000 m$ area, which is represented by a TIN model, built by the application of a Delaunay triangulation on altimetry data of these terrains, retrieved via "http://www.zonums.com/gmaps/terrain.php?action=sample". The parameters of the experiment are listed in Table 1.

Table 1: Simulation setup.

\begin{tabular}{|lll|}
\hline Parameter & Description & Value \\
\hline$V$ & UAV speed & {$[20,90] \mathrm{km} / \mathrm{h}$} \\
$N$ & Number of nodes & {$[100,240]$} \\
$B u f$. & Buffer size & $2 \mathrm{Mbit}$ \\
$N_{m}$ & Number of UAVs & {$[4,11]$} \\
$k$ & Eonstant coefficient & $10^{-4}$ joule $/ \mathrm{m}^{2}$ \\
$c$ & Energy coefficient & 30 joule $/ \mathrm{m}$ \\
\hline
\end{tabular}

We compare IDAS with baseline approaches LEEF [2], CISIL [1], DQNMDC [5] and DRLDC [4] in terms of aggregation ratio, energy cost and maximum energy cost while varying UAV count $\left(N_{m}\right)$, velocity $(V)$, number of nodes $(N)$, and data collection rate $(D c r)$. The terrain parameters are listed in Table 2.

Table 2: Terrain types, risk rates, and elevation.

\begin{tabular}{|l|l|l|}
\hline Type & Risk & Elevation \\
\hline Mountain & 0.004 & $(0,1]$ \\
Forest & 0.002 & $(0,5]$ \\
\hline
\end{tabular}

\subsection{Aggregation Ratio}

As shown in Fig. 1(a), the aggregation ratio grows rapidly at the beginning with $V$ and eventually levels off for all strategies. It is obvious that IDAS achieves the highest aggregation ratio. The reason for that is the DRL based energy efficient UAV route design helps to discover the optimal route such that the trade-off between aggregation ratio and energy cost is achieved. The adverse impact on the aggregation ratio is shown in Fig. 1(b). It is clear that the aggregation ratio drops as $N$ increases and eventually gets stable for all strategies. The reason for that is as follows. The traveling distance increase with more nodes involved such that more data is lost at the beginning. However, such distance grows slowly while the deployment area is densely populated eventually. It is clear that IDAS still performs the best among all.

Fig. 2 suggests the proposed IDAS is more suitable in after disaster scenarios for better data aggregation.

\subsection{Energy Cost}

It can be observed from Fig. 2(a) that each approach consumes more energy as $N$ increases with $N_{m}=11$. It is clearly that IDAS outperforms all baseline approaches. This is because although more nodes are involved, the energy cost is no longer increased rapidly with the help of the optimal route design. As shown in Fig. 2(b), the maximum energy cost for each strategy drops as $N_{m}$ increases. The proposed strategy

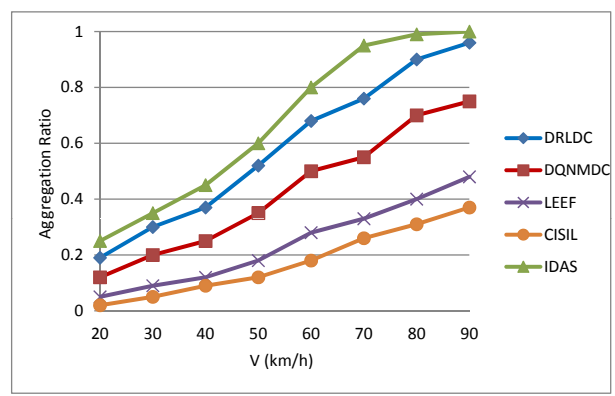

(a)

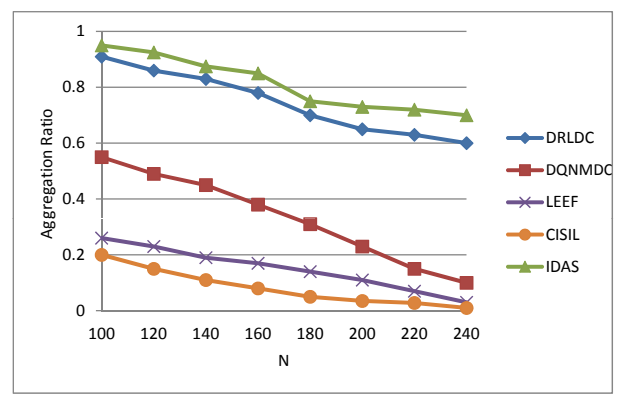

(b)

Figure 1: The aggregation ratio comparison while varying (a) $V$ and (b) $N$

IDAS performs better than others in a relatively lower maximum energy cost. Besides, $N_{m}$ seems to affect IDAS less than other strategies due to the consideration of the trade-off between aggregation ratio and energy cost. Fig. 2(a) and Fig. 2(b) verify the advantage of the proposed IDAS in energy cost for after disaster scenarios.

\section{CONCLUSION}

In this paper, an Intelligent UAV based Data Aggregation Strategy, named (IDAS), is proposed for after disaster scenarios in IoT. Specifically, IDAS consists of an data aggregation task distribution and a deep reinforcement learning based UAV route design, both of which collaborate to achieve the trade-off between the aggregation ratio and the energy cost. The simulation results indicate that IDAS is highly energy efficient while compared with contemporary strategies.

\section{ACKNOWLEDGMENTS}

The authors would like to thank the National Science Foundation of China No. 61702103.

\section{REFERENCES}

[1] W. Lalouani, M. Younis, and N. Badache, "Interconnecting isolated network segments through intermittent links," Journal of Network and Computer Applications, vol. 108, pp. 53-63, 2018.

[2] S. Lee, M. Younis, B. Anglin, and M. Lee, "LEEF: Latency and energy efficient federation of disjoint wireless sensor segments", Ad Hoc Networks, vol. 71, pp. 88-103, 2018. 


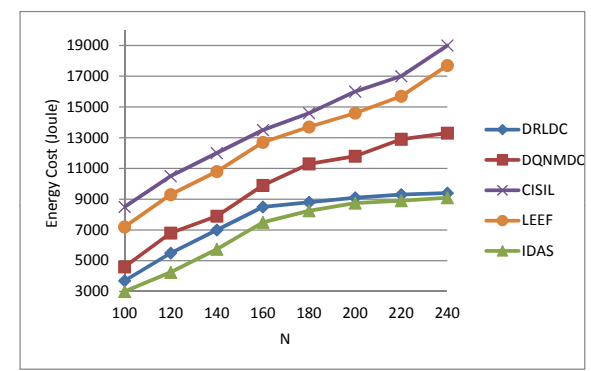

(a)

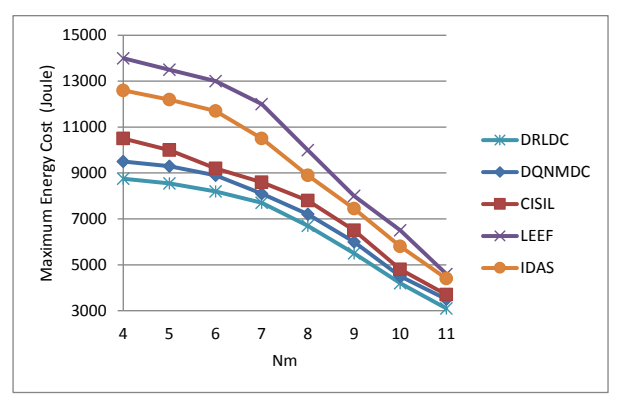

(b)

Figure 2: The energy cost comparison while varying (a) $N$ and (b) $N_{m}$.

[3] Y. K. Joshi, and M. Younis, "Restoring connectivity in a resource constrained WSN," Journal of Network and Computer Applications, vol. 66, pp. 151-165, 2016.

[4] C. H. Liu, Q. Lin, and S. Wen, "Blockchain-enabled data collection and sharing for industrial IoT with deep reinforcement learning," IEEE Transactions on Industrial Informatics, vol. 15, no. 6, pp. 3516-3526, 2018.

[5] K. Toyoshima, T. Oda, M. Hirota, K. Katayama, L.Barolli, "A DQN Based Mobile Actor Node Control in WSAN: Simulation Results of Different Distributions of Events Considering Three-Dimensional Environment," In: International Conference on Emerging Internetworking, Data and Web Technologies. Springer, Cham, pp. 197-209, 2020.

[6] L. Goratti, T. Baykas, T. Rasheed, and S. Kato, "NACRP: A connectivity protocol for star topology wireless sensor networks," IEEE Wireless Communication Letter, vol. 5, no. 2, pp. 120-123 2016.

[7] Z. Xu, L. Chen, C. Chen, and X. Guan, "Joint clustering and routing design for reliable and efficient data collection in largescale wireless sensor networks," IEEE Internet of Things Journal, vol. 3, no. 4, pp. 520-532, 2016.

[8] A. Abbas, and M. Younis, "Establishing connectivity among disjoint terminals using a mix of stationary and mobile relays," Computer Communications, vol. 36, no. 13, pp. 1411-1421, 2013.

[9] X. Wang, L. Xu, S. Zhou, and W. Wu, "Hybrid Recovery Strategy Based on Random Terrain in Wireless Sensor Networks," Scientific Programming, vol. 2017, article id. 5807289, 2017.

[10] I. F. Senturk, K. Akkaya, and S. Janansefat, "Towards realistic connectivity restoration in partitioned mobile sensor networks,' International Journal of Communication Systems, vol. 29, no. 2, pp. 230-250, 2016.

[11] J. Wang, H. Zhang, Z. Ruan, T. Wang, and X. D Wang, "A Machine Learning Based Connectivity Restoration Strategy for Industrial IoTs," IEEE Access, vol. 8, pp. 71136-71145, 2020.

[12] D. Zorbas, L. D. P. Pugliese, T. Razafindralambo, and F. Guerriero, "Optimal drone placement and cost-efficient target coverage," J. Netw. Comput. Appl., vol. 75, pp. 16-31, Nov. 2016.
[13] E. Tuba, R. Capor-Hrosik, A. Alihodzic, and M. Tuba, "Drone place- ment for optimal coverage by brain storm optimization algorithm," in Proc. Int. Conf. Health Inf. Sci. Cham, Switzerland: Springer, 2017, pp. 167-176.

[14] H. Shakhatreh and A. Khreishah, "Optimal Placement of a UAV to maxi- mize the lifetime of wireless devices," 2018, arXiv:1804.02144. [Online]. Available: https://arxiv.org/abs/1804.02144.

[15] A. Rodriguez, A. Gutierrez, L. Rivera, and L. Ramirez "RWA: Comparison of genetic algorithms and simulated annealing in dynamic traffic," in Proc. Adv. Comput. Commun. Eng. Technol. Cham, Switzerland: Springer, 2015, pp. 3-14.

[16] N. Lin, L. Fu, L. Zhao, G. Min, A. Al-Dubai and H. Gacanin, A Novel Multimodal Collaborative Drone-Assisted VANET Networking Model," in IEEE Transactions on Wireless Communications, vol. 19, no. 7, pp. 4919-4933, July 2020. 